\title{
The effects of 2 weeks of interval vs continuous walking training on glycaemic control and whole-body oxidative stress in individuals with type 2 diabetes: a controlled, randomised, crossover trial
}

\author{
Kristian Karstoft $^{1}$ (D) Margaret A. Clark $^{1}$ • Ida Jakobsen ${ }^{1}$ • Ida A. Müller ${ }^{1}$. \\ Bente K. Pedersen $^{1}$ - Thomas P. J. Solomon ${ }^{2,3}$ - Mathias Ried-Larsen ${ }^{1,4}$
}

Received: 2 August 2016 / Accepted: 14 November 2016 / Published online: 9 December 2016

(C) Springer-Verlag Berlin Heidelberg 2016

\begin{abstract}
Aims/hypothesis The aim of this study was to evaluate the effects of oxygen consumption-matched short-term interval walking training (IWT) vs continuous walking training (CWT) on glycaemic control, including glycaemic variability, in individuals with type 2 diabetes. We also assessed whether any training-induced improvements in glycaemic control were associated with systemic oxidative stress levels.

Methods Participants $(n=14)$ with type 2 diabetes completed a crossover trial using three interventions (control intervention [CON], CWT and IWT), each lasting 2 weeks. These were performed in a randomised order (computerised generated randomisation) and separated by washout periods of 4 or 8 weeks after CON or training interventions, respectively. Training included ten supervised treadmill sessions, lasting $60 \mathrm{~min} / \mathrm{session}$, and was performed at the research facility. CWT was performed at moderate walking speed $(75.6 \% \pm 2.5 \%$ of walking peak oxygen consumption $\left.\left[\dot{V} \mathrm{O}_{2 \text { peak }}\right]\right)$, while IWT was performed as alternating $3 \mathrm{~min}$ repetitions at slow $\left(58.9 \% \pm 2.0 \% \dot{V} \mathrm{O}_{2 \text { peak }}\right)$ and fast
\end{abstract}

Kristian Karstoft

k_karstoft@dadlnet.dk

1 The Centre of Inflammation and Metabolism (CIM) and The Centre for Physical Activity Research (CFAS), section M7641,

Rigshospitalet, University of Copenhagen, Blegdamsvej 9,

DK-2100 Copenhagen, Denmark

2 School of Sport, Exercise, and Rehabilitation Sciences, University of Birmingham, Birmingham, UK

3 Institute of Metabolism and Systems Research (IMSR), University of Birmingham, Birmingham, UK

4 The Danish Diabetes Academy, Odense University Hospital, Odense, Denmark $\left(90.0 \% \pm 3.6 \% \dot{V} \mathrm{O}_{2 \text { peak }}\right)$ walking speed. Before and after each intervention, the following was assessed: $24 \mathrm{~h}$ continuous glucose monitoring (CGM) and urinary free 8-iso prostaglandin $\mathrm{F}_{2 \alpha}$ (8-iso $\mathrm{PGF}_{2 \alpha}$; a marker for oxidative stress), physical fitness and body composition. Neither participants nor assessors were blinded to the interventions.

Results No intervention-induced changes were seen in physical fitness or body composition. Compared with baseline, IWT reduced mean glucose levels non-significantly $(-0.7$ $\pm 0.3 \mathrm{mmol} / \mathrm{l}, p=0.08)$ and significantly reduced maximum glucose levels $(-1.8 \pm 0.5 \mathrm{mmol} / 1, p=0.04)$ and mean amplitude of glycaemic excursions (MAGE; $-1.7 \pm 0.4 \mathrm{mmol} / \mathrm{l}$, $p=0.02$ ), whereas no significant within-group changes were seen with CON or CWT. Although 8-iso $\mathrm{PGF}_{2 \alpha}$ was associated with minimum glucose levels at baseline, no change in 8iso $\mathrm{PGF}_{2 \alpha}$ was seen with any intervention, nor were there any associations between changes in 8 -iso $\mathrm{PGF}_{2 \alpha}$ and changes in glycaemic control ( $p>0.05$ for all). No adverse effects were observed with any of the interventions.

Conclusions/interpretation Short-term IWT, but not CWT, improves CGM-derived measures of glycaemic control independent of changes in physical fitness and body composition in individuals with type 2 diabetes. Systemic oxidative stress levels are unaffected by short-term walking and changes in oxidative stress levels are not associated with changes in glycaemic control.

Trial registration ClinicalTrials.gov NCT02320526

Funding The Centre for Physical Activity Research (CFAS) is supported by a grant from TrygFonden. During the study period, the Centre of Inflammation and Metabolism (CIM) was supported by a grant from the Danish National Research Foundation (DNRF55). The study was further supported by grants from Diabetesforeningen, Augustinusfonden and Krista og Viggo Petersens Fond. CIM/CFAS is a member of 
the Danish Center for Strategic Research in Type 2 Diabetes (DD2; the Danish Council for Strategic Research, grant no. 09-067009 and 09-075724). MR-L was supported by a postdoctoral grant from the Danish Diabetes Academy supported by the Novo Nordisk Foundation.

Keywords 8-Isoprostanes - Continuous glucose monitoring · Exercise training - Glycaemic variability $\cdot$ Hypoglycaemia $\cdot$ Lifestyle intervention(s) · Mean amplitude of glycaemic excursions $\cdot$ Physical activity intervention(s) $\cdot$ Systemic inflammation

\begin{tabular}{|c|c|}
\hline \multicolumn{2}{|c|}{ Abbreviations } \\
\hline 8-iso $\mathrm{PGF}_{2 \alpha}$ & Urinary free 8-iso prostaglandin $\mathrm{F}_{2 \alpha}$ \\
\hline $\mathrm{CON}$ & Control intervention \\
\hline CGM & Continuous glucose monitor \\
\hline CWT & Continuous walking training \\
\hline $\mathrm{E} \%$ & Per cent of energy \\
\hline HR & Heart rate \\
\hline hs-CRP & High-sensitive C-reactive protein \\
\hline IWT & Interval walking training \\
\hline MAGE & Mean amplitude of glycaemic excursions \\
\hline RPE & Rate of perceived exertion \\
\hline$\dot{V} \mathrm{O}_{2 \max }$ & Maximal oxygen consumption \\
\hline$\dot{V} \mathrm{O}_{2 \text { peak }}$ & Peak oxygen consumption \\
\hline
\end{tabular}

\section{Introduction}

Regular physical activity is recommended for individuals with type 2 diabetes and the positive effect of physical activity on risk factors for cardiovascular disease in these individuals is well-documented [1]. The exercise structure is a major determinant for the metabolic benefits seen. In this respect, interval training modalities, in which there are alternating periods of high- and low-intensity exercise, have proven effective [2]. As such, we [3] and others [4, 5] have shown that interval training programmes are superior to continuous training programmes matched for total energy expenditure for improving cardiovascular risk factors in individuals with metabolic disease, or equally effective as continuous training despite a lower time and energy consumption [6].

Training-induced improvements in cardiovascular risk factors, including glycaemic control, lipid levels and blood pressure, may be mediated by the training per se, or by improvements in body composition [1]. In support of this, studies comparing training with weight loss vs training without weight loss have found the former to have superior beneficial effects on cardiovascular risk factors $[7,8]$. Since interval training is associated with better improvements in body composition compared with energy expenditure-matched continuous training $[3,9,10]$, it is unclear whether the superior improvements seen in cardiovascular risk factors with interval training are dependent on the training per se, and/or on the differential improvements in body composition. Whether interval training improves glycaemic control independent of changes in body composition is clinically important, as underlined by the minor or nonexistent weight loss reported in meta-analyses evaluating long-term training studies [11, 12].

Compared with healthy individuals, individuals with type 2 diabetes have increased rates of cardiovascular mortality [13], which are suggested to be dependent on increased systemic oxidative stress [14] and associated systemic inflammation in these patients [15]. Poor glycaemic control is associated with increased levels of systemic oxidative stress and inflammation, and it is proposed that glucose instability (i.e. large glycaemic variability) is more closely associated with increased systemic oxidative stress than mean glucose levels $[16,17]$. Moreover, while increased mean blood glucose levels are predictive of microvascular disease [18], increased glycaemic variability is more predictive of macrovascular complications [19]. Short-term training programmes have previously been found to reduce glycaemic variability [20, 21]. Whether these reductions in glycaemic variability differ between interval and continuous training programmes and whether such reductions in glycaemic variability translate to reduced levels of systemic oxidative stress is unknown.

Thus, the aim of this study was to assess the effects of interval training vs continuous training on glycaemic control, lipid levels and blood pressure in individuals with type 2 diabetes, independent of changes in body composition. Moreover, we further aimed to evaluate whether potential improvements in glycaemic variability and other measures of glycaemic control were associated with decreased levels of oxidative stress. We tested the hypotheses that compared with continuous training, interval training induces greater improvements in glycaemic control, including reduced glycaemic variability, independent of changes in body composition, and that these improvements are associated with decreased systemic oxidative stress.

\section{Methods}

Participants Individuals with type 2 diabetes [22], with a BMI $>18 \mathrm{~kg} / \mathrm{m}^{2}$ but $<40 \mathrm{~kg} / \mathrm{m}^{2}$, were recruited onto the study. Exclusion criteria were insulin treatment, smoking, pregnancy, renal failure (estimated GFR $<60 \mathrm{ml} / \mathrm{min}$ ), contraindications to increased levels of physical activity [23] and more than moderate levels of maximally moderate-intensity physical activity (>90 min/week). All participants underwent a screening visit consisting of a medical interview and examination, completion of a habitual physical activity questionnaire [24], a walking test for peak oxygen consumption 
$\left(\dot{V} \mathrm{O}_{2 \text { peak }}\right)$, with continuous oxygen uptake measurements (Cosmed K4B2; Cosmed, Rome, Italy), and a familiarisation test for maximal oxygen consumption $\left(\dot{V} \mathrm{O}_{2 \max }\right)$. Participants gave informed consent before any investigational procedures were performed. Baseline characteristics are given in the Results section. The study was approved by the regional ethical committee (H-6-2014-043) and is a registered trial (ClinicalTrials.gov registration no. NCT02320526).

Study design Participants were included in a crossover study consisting of three interventions, each lasting 2 weeks, with investigations being performed before and after each intervention. Between interventions, washout periods (8 weeks after training interventions, 4 weeks after control intervention [CON]) were applied, wherein participants returned to their habitual activity levels. Interventions were performed in a randomised order (computer generated randomisation was carried out using www.randomization.com accessed 6 Nov 2014 [for reproduction use seed 18512]) and participants were informed about the upcoming intervention at the end of the pre-intervention investigation.

Interventions The training interventions consisted of ten fully supervised 60 min walking sessions on a treadmill (Katana; Lode, Groningen, the Netherlands). Training was performed 5 days per week (every weekday) but not during the weekend. Continuous walking training (CWT) consisted of continuous walking with a speed aiming for $\sim 73 \%$ of $\dot{V} \mathrm{O}_{2 \text { peak, }}$, whereas interval walking training (IWT) consisted of alternating cycles of 3 min slow walking ( $54 \%$ of $\dot{V} \mathrm{O}_{2 \text { peak }}$ ) and 3 min fast walking ( $89 \%$ of $\left.\dot{V} \mathrm{O}_{2 \text { peak }}\right)$. These intensities have previously been found sustainable in a free-living training study [3]. Adequate walking speeds to reach the desired intensities were determined by performing continuous measurements of oxygen uptake (Cosmed Quark; Cosmed) during the first and sixth walking sessions in each training intervention. These walking speeds were then repeated for the following four exercise sessions. All sessions were performed with a heart rate (HR) monitor (Polar RC3; Polar, Kempele, Finland) and the rate of perceived exertion (RPE) was reported immediately after each session [25]. For $\mathrm{CON}$, participants were instructed to continue their life without any changes.

Investigations Investigations were performed over 4 days (days 0-3) immediately before (pre) and after (post) each intervention. On day 0 , participants reported to the laboratory for initiation of continuous glucose monitoring and for provision of a standardised diet. A continuous glucose monitor (CGM) glucose sensor (Enlite; Medtronic, Fridley, MN, USA) was inserted into the abdominal subcutaneous adipose tissue and connected to a CGM monitor (iPro2; Medtronic). The CGM was calibrated four times each day (before main meals and at bedtime) by a glucose meter (Contour; Bayer, Basel, Switzerland). Participants went home after being instructed to avoid strenuous physical activity, alcohol and caffeine during the next 3 days. Otherwise, they were instructed to live as representatively to their normal life as possible.

On day 1, participants consumed a self-selected and 'typical diet' (based on each individual), which was registered in a diet record. This diet record was distributed to the participants so that they could duplicate the chosen diet on day 1 of subsequent interventions.

On day 2, participants only consumed a provided, standardised diet; food was divided into three equal portions, consumed by participants at 07:00, 13:00 and 19:00 hours. The diet was intended to be isoenergetic and was based on the Mifflin St Jeor Equation multiplied by a physical activity level of 1.4 metabolic equivalents (METS) [26]. The diet consisted of a nutritional drink (Resource Komplett 1.5; Nestlé, Vevey, Switzerland) with a mixed macronutrient composition (15\% of energy [E\%] protein, $55 \mathrm{E} \%$ carbohydrate, $30 \mathrm{E} \%$ fat). Moreover, participants collected $24 \mathrm{~h}$ urine in a plastic container, starting with an empty bladder at 07:00 hours. The plastic container was kept in a cooling bag throughout the urine collection period.

On day 3, participants reported to the laboratory at 07:00 hours, after having fasted ( $\geq 8 \mathrm{~h}$, water excluded). The CGM system was removed and, after a final void, the urine container was collected. A blood sample was taken and body mass was assessed. After supine resting, participants were offered a small meal, following which a dual-energy x-ray absorptiometry (DXA) scan (Lunar Prodigy Advance; GE Healthcare, Madison, WI, USA) was performed followed by blood pressure measurements (Microlife BP meter; Microlife, Widnau, Switzerland). Finally, a $\dot{V} \mathrm{O}_{2 \max }$ test was performed using a previously described protocol [3].

The post-intervention investigations were planned so that the last IWT/CWT training session was performed on day 1, between 12:00 hours and 16:00 hours (15-19 h before initiation of urine collection). To avoid energy deficit during the IWT/CWT post-intervention investigations, participants were given a snack (200 ml nutritional drink, $1255 \mathrm{~kJ}$; Resource Komplett 1.5) immediately after the last IWT/CWT session.

Blood and urine sample analyses Blood samples for glucose measurements were collected in heparinised syringes and analysed at a bedside platform (ABL 700; Radiometer, Herlev, Denmark). For analysis of $\mathrm{HbA}_{1 \mathrm{c}}$ and TNF- $\alpha$ and IFN- $\gamma$ (proinflammatory cytokines), samples were collected in EDTA-coated tubes, whereas for high-sensitive C-reactive protein (hs-CRP), insulin and lipids, samples were collected in lithium-heparin-coated tubes. All samples were analysed immediately, except for those for proinflammatory cytokine measurements which were centrifuged $\left(2000 \mathrm{~g}, 4^{\circ} \mathrm{C}, 15 \mathrm{~min}\right)$ and stored at $-80^{\circ} \mathrm{C}$ until analysis. 
$\mathrm{HbA}_{1 \mathrm{c}}$ was analysed using absorption photometry (Tosoh G7; Tosoh, San Francisco, CA, USA), insulin was analysed using Electrochemiluminescence immunoassay (Cobas 8000, Roche Diagnostics, IN, USA) and lipids were analysed using absorption photometry (Cobas 8000, Roche Diagnostics). Proinflammatory cytokines were analysed in duplicate, using the V-plex pro-inflammatory panel (MSD, Rockville, MD, USA).

Urine volume was measured and urine aliquots were frozen at $-80^{\circ} \mathrm{C}$ until analysis. Urinary creatinine was measured via absorption photometry (Cobas 8000, Roche Diagnostics) and urinary free 8-iso prostaglandin $\mathrm{F}_{2 \alpha}$ (8-iso $\mathrm{PGF}_{2 \alpha}$ ), a marker for oxidative stress [16], was assessed in triplicate using the 8Isoprostane ELISA Kit (Cayman Chemicals, Ann Arbor, MI, USA).

Calculations Mean rates of oxygen uptake were calculated during the first and the sixth IWT/CWT session. Moreover, the mean rates of oxygen uptake from the last minute of each slow and fast IWT interval were calculated during the same IWT sessions. Mean HR was calculated from all IWT/CWT sessions and from the last minute of slow and fast IWT intervals.

Twenty-four-hour CGM measurements, aligned with the urine collection period (from 07:00 hours on day 2 to 07:00 hours on day 3 ), were analysed for mean, minimum and maximum glucose levels, time fraction spent in hyperglycaemia (>10.0 mmol/l) and hypoglycaemia (<4.0 mmol/l, [27]), and mean amplitude of glycaemic excursions (MAGE; a marker for glycaemic variability [28]). The 8-iso $\mathrm{PGF}_{2 \alpha}$ level was normalised to urinary creatinine excretion.

Statistics Pre-intervention variables were compared using oneway repeated-measures ANOVA. Training variables (IWT vs CWT) were compared using the Student's paired $t$ test. The adequacy of the washout periods was assessed using the Student's paired $t$ test, comparing pre-intervention variables of a given intervention with the pre-intervention variables of the following intervention. Intervention-induced differences within interventions were assessed using two-way repeatedmeasures (intervention $\times$ time) ANOVA. Intervention-induced differences between interventions were assessed using one-way repeated-measures ANOVA of the $\Delta$ values for each intervention (post - pre-intervention). For all ANOVA analyses, Bonferroni post hoc tests were applied to assess differences within/between interventions.

Associations between 8 -iso $\mathrm{PGF}_{2 \alpha}$ and measures of glycaemic control (both baseline and $\Delta$ [change] values) were analysed separately for each intervention using linear regression models. Since the strength and direction of the associations were uniform between interventions, all values were pooled to increase statistical power. For the pooled analyses, we used random intercept models including participants as random effects. Moreover, the associations between changes were adjusted for the baseline values of 8-iso $\mathrm{PGF}_{2 \alpha}$ and measures of glycaemic control, to account for regression towards the mean. Residuals were checked for normality and homoscedasticity.

All participants completed all investigational procedures but due to a CGM sensor failure in one individual (CWT post-intervention), ANOVA analyses of the CGM data only includes 13 individuals.

All statistical analyses were two-tailed and performed using Prism v6.03 (GraphPad Software, San Diego, CA, USA) or Stata v13.1 (Stata Corporation, College Station, TX, USA). Data are reported as mean \pm SEM unless indicated otherwise. $p$ values $<0.05$ were considered significant.

\section{Results}

Participant characteristics Fourteen participants (11 men; aged $65 \pm 2$ years; years since diabetes diagnosis, $9 \pm 1$; leisure time physical activity, $1201 \pm 226 \mathrm{~kJ} /$ day) were included in the study. All participants continued their usual glucose-lowering medication during the study period (metformin $[n=14]$, sulfonylureas $[n=3$ ], glucagon-like peptide-1 [GLP-1] analogues $[n=3])$, with no changes in dose or type. Mean dietary energy intake (apart from the extra $1255 \mathrm{~kJ}$ on day 1 during the training interventions) was $8339 \pm 674$ and $10,117 \pm 418 \mathrm{~kJ}$ on day 1 and day 2 , respectively, with no differences within or between interventions (data not shown).

Interventions Two participants missed a single training session in both IWT and CWT resulting in a similar training adherence (sessions completed relative to sessions prescribed) of $99 \%$ in both interventions.

There was no difference in mean rate of oxygen uptake between CWT $\left(1524 \pm 78 \mathrm{ml} / \mathrm{min}\left[75.6 \% \pm 2.5 \%\right.\right.$ of $\left.\left.\dot{V} \mathrm{O}_{2 \text { peak }}\right]\right)$ and IWT $\left(1513 \pm 78 \mathrm{ml} / \mathrm{min}\left[75.0 \% \pm 2.5 \%\right.\right.$ of $\left.\left.\dot{V} \mathrm{O}_{2 \text { peak }}\right], p>0.05\right)$. Compared with the rate of oxygen uptake during CWT, the rate was lower during low-intensity walking periods in IWT $\left(1190 \pm 62 \mathrm{ml} / \mathrm{min}\left[58.9 \% \pm 2.0 \%\right.\right.$ of $\left.\left.\dot{V} \mathrm{O}_{2 \text { peak }}\right]\right)$ and higher during high-intensity walking periods in IWT $(1814 \pm 99 \mathrm{ml} / \mathrm{min}$ [90.0\% $\pm 3.6 \%$ of $\left.\left.\dot{V} \mathrm{O}_{2 \text { peak }}\right]\right)(p<0.001$ for both).

Mean HR was $108 \pm 3 \mathrm{bpm}$ during CWT and $109 \pm 3 \mathrm{bpm}$ during IWT $(p>0.05)$, with the HR during low-intensity walking periods in IWT $(100 \pm 2 \mathrm{bpm})$ being lower, and that during high-intensity walking periods in IWT $(119 \pm 3)$ being higher than the overall CWT HR ( $p<0.001$ for both).

Mean walking speed was faster during CWT $(5.0 \pm 0.1 \mathrm{~km} / \mathrm{h})$ than during IWT $(4.7 \pm 0.1 \mathrm{~km} / \mathrm{h}, p<0.001)$; however, the slow IWT walking speed $(3.4 \pm 0.1 \mathrm{~km} / \mathrm{h})$ was slower and fast IWT walking speed $(6.0 \pm 0.1 \mathrm{~km} / \mathrm{h})$ was faster than CWT walking speed ( $p<0.001$ for both). Moreover, mean walking speed increased from the first to the sixth 
training session in both CWT $(4.8 \pm 0.1$ vs $5.0 \pm 0.1 \mathrm{~km} / \mathrm{h}$, $p=0.001)$ and IWT ( $4.6 \pm 0.1$ vs $4.8 \pm 0.1 \mathrm{~km} / \mathrm{h}, p=0.01)$.

Mean RPE was lower in CWT (11.1 \pm 0.5 arbitrary units) than in IWT $(12.5 \pm 0.4$ arbitrary units, $p=0.002)$.

Physical fitness and body composition No pre-intervention differences were seen in any variables related to physical fitness or body composition ( $p>0.05$ for all comparisons; Table 1). No changes in physical fitness (either absolute or relative to body weight) were observed with any intervention, nor were there any intervention-induced differences between interventions $(p>0.05$ for all comparisons). Body mass was non-significantly decreased after the CWT intervention $(-0.4 \pm 0.2 \mathrm{~kg}, p=0.09)$. Neither CON nor IWT resulted in changes in body mass and no intervention-induced differences between interventions were seen. For all other body composition assessments (fat-free mass and total, android and gynoid fat percentage), no interventioninduced changes and no intervention-induced differences between interventions were seen $(p>0.05$ for all comparisons; Table 1).

Glycaemic control No pre-intervention differences were seen in any glycaemic control variables ( $p>0.05$ for all comparisons) (Fig. 1, Table 1). No significant changes in $\mathrm{HbA}_{1 \mathrm{c}}$ or fasting glucose were seen with any of the interventions, and no intervention-induced differences between interventions were seen for $\mathrm{HbA}_{1 \mathrm{c}}$ ( $p>0.05$ for all comparisons). Regarding fasting glucose, IWT resulted in a significant reduction compared with CON $(-0.5 \pm 0.2$ vs $0.2 \pm 0.3 \mathrm{mmol} / \mathrm{l}$, $p=0.04$; Table 1).

Mean CGM glucose levels and time spent in hyperglycaemia did not change after CON or CWT interventions $(p>0.05)$ but showed a trend towards decrease after IWT $(-0.7 \pm 0.3 \mathrm{mmol} / \mathrm{l}$, $p=0.08$ and $-9.5 \pm 4.1 \%$ of time, $p=0.08$, respectively; Fig. 1a,e and Table 1). Maximum CGM glucose levels and MAGE did not change after CON or CWT interventions $(p>0.05)$ but were decreased following IWT $(-1.8 \pm 0.5 \mathrm{mmol} / \mathrm{l}, p=0.04$ and -1.7 $\pm 0.4 \mathrm{mmol} / \mathrm{l}, p=0.02$, respectively; Fig. $1 \mathrm{c}$,f and Table 1). Moreover, IWT resulted in significant reductions in mean and maximum CGM glucose levels and time spent in hyperglycaemia compared with CON (CON: $0.5 \pm 0.3 \mathrm{mmol} / \mathrm{l}$ $[p=0.04], 1.6 \pm 0.7 \mathrm{mmol} / 1[p=0.01]$ and $8.5 \pm 3.9 \%$ of time $[p=0.03]$, respectively; Table 1$)$. No changes in minimum CGM glucose levels or time spent in hypoglycaemia were seen with any of the interventions, neither were there any intervention-induced differences between interventions ( $p>0.05$ for all comparisons; Fig. 1b,d and Table 1).

Lipids and blood pressure No pre-intervention differences were seen in blood pressure or any lipid variables ( $p>0.05$ for all comparisons; Table 1). IWT reduced total cholesterols $(-0.3 \pm 0.1 \mathrm{mmol} / \mathrm{l}, p=0.03)$ and triacylglycerol $(-0.2 \pm 0.1 \mathrm{mmol} / \mathrm{l}, p=0.02)$, with no effects of CWT or
CON on any lipid variables ( $p>0.05$ for all comparisons). Moreover, no intervention-induced differences in any lipid variables were seen between any of the interventions ( $p>0.05$ for all comparisons). No change in blood pressure (either systolic or diastolic) was seen with any of the interventions, neither were there any intervention-induced differences between interventions $(p>0.05$ for all comparisons).

Systemic oxidative stress and inflammation No preintervention differences were seen in levels of 8-iso $\mathrm{PGF}_{2 \alpha}$, hs-CRP, TNF- $\alpha$ or IFN- $\gamma$ ( $p>0.05$ for all comparisons; Table 1). Moreover, no changes in any of these variables were seen with any of the interventions, neither were there any intervention-induced differences between interventions ( $p>0.05$ for all comparisons).

Washout periods Total cholesterol levels were nonsignificantly higher before IWT than before the following intervention $(4.3 \pm 0.4 \mathrm{mmol} / 1$ vs $4.1 \pm 0.4 \mathrm{mmol} / \mathrm{l}, p=0.06)$. For all other clinical variables mentioned in Table 1 , no differences were seen between pre-IWT/CWT values and preintervention values of the following intervention.

Associations between 8-iso $\mathbf{P G F}_{2 \alpha}$ and glycaemic control Mean and maximum CGM glucose levels, time spent in hyperglycaemia and MAGE were not associated with urinary 8-iso PGF $_{2 \alpha}$ at baseline (Table 2). In contrast, minimum CGM glucose levels were negatively associated with 8 -iso $\mathrm{PGF}_{2 \alpha}$ (Fig. 2a and Table 2) and time spent in hypoglycaemia was positively associated with 8 -iso $\mathrm{PGF}_{2 \alpha}$ (Fig. $2 \mathrm{~b}$ and Table 2). These associations were consistent after Bonferroni correction of the $p$ values.

No significant associations between changes in 8-iso $\mathrm{PGF}_{2 \alpha}$ and changes in measures of glycaemic control were found (Table 2). Adjustments for the baseline levels of 8-iso $\mathrm{PGF}_{2 \alpha}$ and measures of glycaemic control did not influence the associations (data not shown). The association between changes in 8-iso $\mathrm{PGF}_{2 \alpha}$ and changes in maximum CGM glucose was borderline significant $\left(p=0.06, r^{2}=0.09\right.$; Table 2) but only before Bonferroni correction.

\section{Discussion}

The main finding from this study is that IWT, but not time duration- and oxygen consumption-matched CWT, improves CGM-derived measures of glycaemic control including glycaemic variability, independent of changes in body composition and physical fitness in individuals with type 2 diabetes. This highlights the importance of training pattern in exercise-induced metabolic improvements and suggests that the superior effects previously reported after IWT compared 
Table 1 Clinical variables

\begin{tabular}{|c|c|c|c|c|c|c|c|}
\hline \multirow[t]{2}{*}{ Variable } & \multicolumn{2}{|l|}{$\mathrm{CON}$} & \multicolumn{2}{|l|}{ CWT } & \multicolumn{2}{|l|}{ IWT } & \multirow{2}{*}{$\begin{array}{l}\text { CON vs IWT } \\
(p \text { value })\end{array}$} \\
\hline & Pre & Post & Pre & Post & Pre & Post & \\
\hline \multicolumn{8}{|l|}{ Physical fitness } \\
\hline Absolute $\dot{V} \mathrm{O}_{2 \max }(\mathrm{l} / \mathrm{min})$ & $2.25 \pm 0.18$ & $2.24 \pm 0.14$ & $2.31 \pm 0.15$ & $2.31 \pm 0.15$ & $2.30 \pm 0.16$ & $2.34 \pm 0.15$ & \\
\hline Relative $\dot{V} \mathrm{O}_{2 \max }\left(\mathrm{ml} \mathrm{min}^{-1} \mathrm{~kg}^{-1}\right)$ & $23.8 \pm 1.4$ & $23.8 \pm 1.3$ & $24.6 \pm 1.6$ & $24.8 \pm 1.6$ & $24.5 \pm 1.5$ & $24.7 \pm 1.1$ & \\
\hline \multicolumn{8}{|l|}{ Body composition } \\
\hline Body mass (kg) & $96.8 \pm 4.7$ & $96.7 \pm 4.7$ & $97.7 \pm 4.6$ & $97.3 \pm 4.6^{\S}$ & $97.0 \pm 4.6$ & $96.9 \pm 4.6$ & \\
\hline Fat-free mass (kg) & $61.1 \pm 3.3$ & $61.2 \pm 3.2$ & $61.6 \pm 3.2$ & $61.3 \pm 3.1$ & $61.4 \pm 3.2$ & $61.7 \pm 3.2$ & \\
\hline Total body fat (\%) & $38.3 \pm 1.8$ & $38.1 \pm 1.7$ & $38.3 \pm 1.6$ & $38.0 \pm 1.6$ & $37.8 \pm 1.6$ & $37.7 \pm 1.6$ & \\
\hline Android fat $(\%)$ & $48.6 \pm 1.6$ & $48.2 \pm 1.5$ & $48.5 \pm 1.3$ & $48.5 \pm 1.4$ & $48.1 \pm 1.2$ & $47.7 \pm 1.4$ & \\
\hline Gynoid fat (\%) & $37.4 \pm 2.1$ & $37.4 \pm 2.2$ & $37.6 \pm 1.9$ & $37.8 \pm 2.0$ & $37.2 \pm 2.0$ & $37.0 \pm 2.0$ & \\
\hline \multicolumn{8}{|l|}{ Lipids (mmol/l) } \\
\hline Total cholesterol & $4.1 \pm 0.3$ & $4.1 \pm 0.3$ & $4.2 \pm 0.3$ & $4.0 \pm 0.3$ & $4.4 \pm 0.3$ & $4.1 \pm 0.3 *$ & \\
\hline HDL-cholesterol & $1.1 \pm 0.1$ & $1.0 \pm 0.1$ & $1.1 \pm 0.1$ & $1.1 \pm 0.1$ & $1.1 \pm 0.1$ & $1.1 \pm 0.1$ & \\
\hline LDL-cholesterol & $2.6 \pm 0.3$ & $2.6 \pm 0.3$ & $2.6 \pm 0.3$ & $2.5 \pm 0.3$ & $2.7 \pm 0.3$ & $2.6 \pm 0.3$ & \\
\hline Triacylglycerol $^{\ddagger}$ & $1.6 \pm 0.2$ & $1.5 \pm 0.2$ & $1.5 \pm 0.2$ & $1.4 \pm 0.2$ & $1.6 \pm 0.2$ & $1.4 \pm 0.1 *$ & \\
\hline \multicolumn{8}{|l|}{ Blood pressure (mmHg) } \\
\hline Systolic & $139 \pm 3$ & $142 \pm 4$ & $137 \pm 4$ & $137 \pm 4$ & $142 \pm 5$ & $136 \pm 4$ & \\
\hline Diastolic & $82 \pm 2$ & $84 \pm 2$ & $82 \pm 3$ & $84 \pm 2$ & $84 \pm 3$ & $79 \pm 2$ & \\
\hline \multicolumn{8}{|l|}{ Glycaemic control } \\
\hline $\mathrm{HbA}_{1 \mathrm{c}}(\%)$ & $6.6 \pm 0.3$ & $6.6 \pm 0.3$ & $6.7 \pm 0.3$ & $6.6 \pm 0.4$ & $6.6 \pm 0.3$ & $6.5 \pm 0.3$ & \\
\hline $\mathrm{HbA}_{1 \mathrm{c}}(\mathrm{mmol} / \mathrm{mol})$ & $48.8 \pm 2.4$ & $48.5 \pm 2.5$ & $49.2 \pm 2.4$ & $48.9 \pm 2.6$ & $48.6 \pm 2.5$ & $48.0 \pm 2.5$ & \\
\hline Fasting glucose $(\mathrm{mmol} / \mathrm{l})$ & $7.2 \pm 0.4$ & $7.4 \pm 0.4$ & $7.4 \pm 0.5$ & $7.1 \pm 0.3$ & $7.8 \pm 0.4$ & $7.3 \pm 0.4$ & 0.04 \\
\hline Fasting insulin (pmol/l) & $106 \pm 12$ & $105 \pm 12$ & $96 \pm 10$ & $94 \pm 15$ & $104 \pm 16$ & $98 \pm 13$ & \\
\hline \multicolumn{8}{|l|}{ CGM glucose over $24 \mathrm{~h}$} \\
\hline Mean $(\mathrm{mmol} / \mathrm{l})^{\dagger}$ & $7.6 \pm 0.4$ & $8.1 \pm 0.6$ & $8.0 \pm 0.5$ & $7.5 \pm 0.5$ & $8.4 \pm 0.6$ & $7.7 \pm 0.5^{\S}$ & 0.04 \\
\hline Minimum (mmol/1) & $5.0 \pm 0.3$ & $4.7 \pm 0.5$ & $4.8 \pm 0.3$ & $4.5 \pm 0.4$ & $5.2 \pm 0.5$ & $4.9 \pm 0.4$ & \\
\hline $\operatorname{Maximum}(\mathrm{mmol} / \mathrm{l}) \dagger$ & $12.4 \pm 0.6$ & $14.0 \pm 1.0$ & $13.4 \pm 0.9$ & $13.6 \pm 0.9$ & $14.1 \pm 0.8$ & $12.3 \pm 0.6^{*}$ & 0.01 \\
\hline Glucose $<4.0 \mathrm{mmol} / 1$ ( $\%$ of time $)$ & $1.4 \pm 0.9$ & $2.8 \pm 1.2$ & $3.3 \pm 2.1$ & $5.3 \pm 2.2$ & $4.2 \pm 1.9$ & $4.8 \pm 2.1$ & \\
\hline Glucose $>10.0 \mathrm{mmol} / 1(\% \text { of time })^{\dagger}$ & $15.7 \pm 4.2$ & $24.2 \pm 6.5$ & $21.8 \pm 5.2$ & $18.6 \pm 5.1$ & $28.1 \pm 6.8$ & $18.6 \pm 4.9^{\S}$ & 0.03 \\
\hline $\operatorname{MAGE}(\mathrm{mmol} / \mathrm{l}) \dagger$ & $5.2 \pm 0.4$ & $6.4 \pm 0.6$ & $6.5 \pm 0.7$ & $6.5 \pm 0.7$ & $7.1 \pm 0.6$ & $5.4 \pm 0.4 *$ & 0.01 \\
\hline \multicolumn{8}{|l|}{ Systemic inflammation } \\
\hline hs-CRP (nmol/l) & $15.6 \pm 3.9$ & $15.6 \pm 3.1$ & $23.1 \pm 5.9$ & $21.8 \pm 5.3$ & $15.0 \pm 2.8$ & $16.3 \pm 3.4$ & \\
\hline $\mathrm{IFN}-\gamma(\mathrm{pg} / \mathrm{ml})$ & $3.3 \pm 0.4$ & $4.0 \pm 0.9$ & $3.4 \pm 0.6$ & $3.7 \pm 0.5$ & $3.4 \pm 0.6$ & $3.8 \pm 0.9$ & \\
\hline $\mathrm{TNF}-\alpha(\mathrm{pg} / \mathrm{ml})$ & $2.9 \pm 0.2$ & $2.9 \pm 0.2$ & $3.1 \pm 0.2$ & $3.1 \pm 0.3$ & $2.9 \pm 0.2$ & $2.9 \pm 0.2$ & \\
\hline \multicolumn{8}{|l|}{ Systemic oxidative stress } \\
\hline 8-iso $\mathrm{PGF}_{2 \alpha}(\mathrm{pg} / \mathrm{mg}$ creatinine) & $1007 \pm 65$ & $1047 \pm 94$ & $1148 \pm 127$ & $1051 \pm 114$ & $1257 \pm 152$ & $1090 \pm 84$ & \\
\hline
\end{tabular}

Data are mean \pm SEM

${ }^{*} p<0.05$ and ${ }^{\S} p<0.10$ for within-group pre vs post; ${ }^{\dagger} p<0.05$ for intervention $\times$ time interaction; ${ }^{\star} p<0.05$ for main effect of time

with CWT are not merely mediated by the differential effects on body composition and/or physical fitness [3]. Another important observation from the study is that systemic oxidative stress levels were not changed by any of the interventions and that none of the improvements seen in glycaemic control after IWT translated to reduced levels of oxidative stress.

Although covariate analyses have previously indicated that changes in body composition can only explain part of IWTinduced improvements in metabolic variables [29], studies have found that training interventions with weight loss improve glycaemic control more than training interventions without weight loss $[7 ; 8]$. In this respect, it is interesting that the IWT-induced improvements in glycaemic control seen in the current study are fairly similar to those seen in our previous study evaluating a 4-month intervention, which also induced robust improvements in body composition and physical fitness [3]. Thus, the current study shows that the effects of IWT on glycaemic control are largely explained by factors 
a
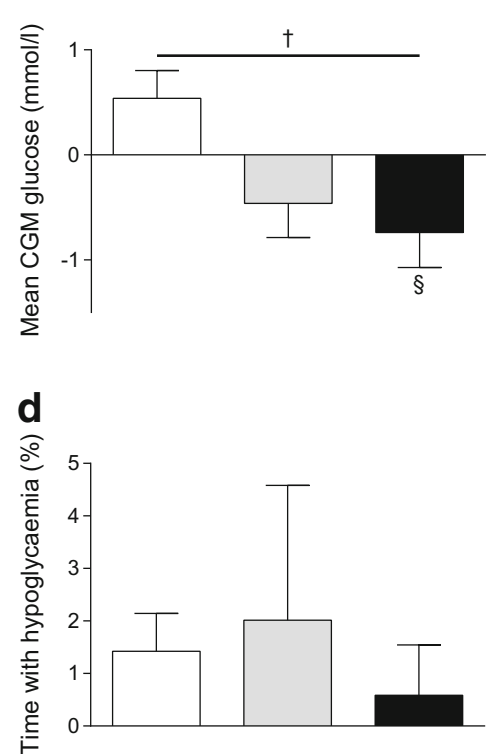

Fig. 1 Changes (post - pre-intervention) in CGM variables measured over 24 h. (a) Mean glucose. (b) Minimum (min.) glucose. (c) Maximum (max.) glucose. (d) Time spent in hypoglycaemia (glucose $<4.0 \mathrm{mmol} / \mathrm{l}, \%$ of time). (e) Time spent in hyperglycaemia (glucose $>10.0 \mathrm{mmol} / 1, \%$ of time). (f) MAGE. White bars, CON; grey bars,

other than IWT-associated improvements in body composition and/or physical fitness. Since the last bout of exercise was performed $15-19 \mathrm{~h}$ before initiation of the CGM measurement period, and since acute exercise may influence insulin sensitivity and therefore glycaemic control for up to $48 \mathrm{~h}$ [30], it cannot be concluded from the current study whether the improvements seen are training-induced or are dependent on the effects of acute exercise. However, since a single IWT
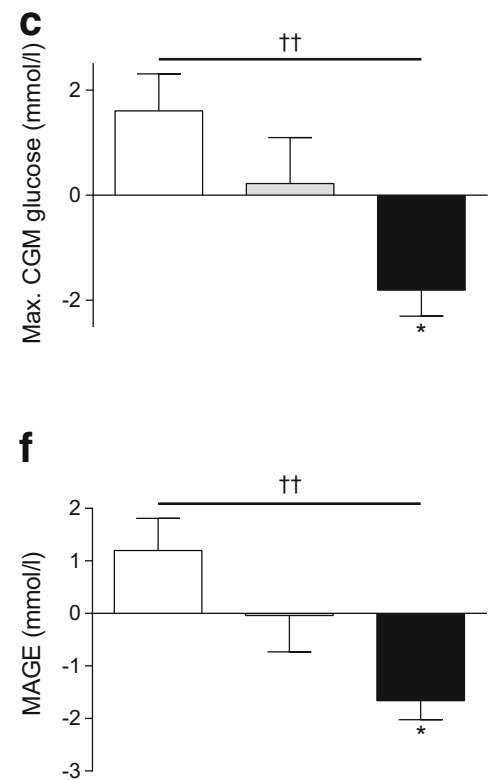

CWT; black bars, IWT. Data are presented as mean \pm SEM. Statistical differences were analysed by two-way repeated measures ANOVA when comparing changes within groups, ${ }^{*} p<0.05$ and $\S p=0.08$; one-way repeated-measures ANOVA of $\Delta$ values when comparing differences between groups, $\uparrow p<0.05, \uparrow \uparrow p<0.01$

session does not differentially influence glycaemic control during the day after an exercise bout compared with a single CWT session [31], and because CWT did not improve any of the glycaemic control variables in the current study, we believe that the training-induced improvements mediate the effects on glycaemic control.

The lack of improvement in metabolic variables with CWT is notable. Whereas we have previously found similar results

Table 2 Association between markers of glycaemic control and urinary 8 -iso $\mathrm{PGF}_{2 \alpha}$

\begin{tabular}{|c|c|c|c|c|}
\hline Marker & $B$ value & $95 \% \mathrm{CI}$ & $p$ value & $r^{2}$ \\
\hline \multicolumn{5}{|l|}{ Baseline } \\
\hline \multicolumn{5}{|l|}{ Urinary 8 -iso $\mathrm{PGF}_{2 \alpha}(\mathrm{pg} / \mathrm{mg}$ creatinine $)$} \\
\hline Mean glucose $(\mathrm{mmol} / \mathrm{l})$ & -39.7 & $-115.4,36.1$ & 0.31 & 0.15 \\
\hline Minimum glucose $(\mathrm{mmol} / \mathrm{l})$ & -156.1 & $-238.5,-73.7$ & $<0.001$ & 0.38 \\
\hline Maximum glucose $(\mathrm{mmol} / \mathrm{l})$ & -0.2 & $-30.1,29.7$ & 0.99 & $<0.01$ \\
\hline Glucose $<4.0 \mathrm{mmol} / 1$ ( $\%$ of time $)$ & 32.4 & $11.3,53.4$ & 0.003 & 0.32 \\
\hline Glucose $>10.0 \mathrm{mmol} / 1$ (\% of time) & -4.2 & $-11.4,3.0$ & 0.25 & 0.14 \\
\hline $\operatorname{MAGE}(\mathrm{mmol} / \mathrm{l})$ & 34.4 & $-19.9,88.7$ & 0.21 & 0.08 \\
\hline \multicolumn{5}{|l|}{$\Delta$ (Change) } \\
\hline \multicolumn{5}{|l|}{$\Delta$ Urinary 8 -iso $\mathrm{PGF}_{2 \alpha}(\mathrm{pg} / \mathrm{mg}$ creatinine $)$} \\
\hline$\Delta$ Mean glucose $(\mathrm{mmol} / \mathrm{l})$ & 28.7 & $-52.3,109.8$ & 0.49 & 0.01 \\
\hline$\Delta$ Minimum glucose $(\mathrm{mmol} / \mathrm{l})$ & -27.4 & $-122.3,67.4$ & 0.57 & 0.01 \\
\hline$\Delta$ Maximum glucose $(\mathrm{mmol} / \mathrm{l})$ & 32.9 & $-1.7,67.5$ & 0.06 & 0.09 \\
\hline$\Delta$ Glucose $<4.0 \mathrm{mmol} / 1$ ( $\%$ of time) & 8.0 & $-11.8,27.9$ & 0.43 & 0.02 \\
\hline$\Delta$ Glucose $>10.0 \mathrm{mmol} / 1$ ( $\%$ of time $)$ & 2.6 & $-3.7,8.8$ & 0.42 & 0.02 \\
\hline$\Delta \mathrm{MAGE}(\mathrm{mmol} / \mathrm{l})$ & 31.5 & $-10.0,73.1$ & 0.14 & 0.06 \\
\hline
\end{tabular}

Associations were assessed using linear regression analyses. Crude $p$ values are provided 
Fig. 2 The association between (a) baseline levels of urinary 8-iso $\mathrm{PGF}_{2 \alpha}$ and minimum blood glucose and (b) baseline concentrations of urinary 8 -iso $\mathrm{PGF}_{2 \alpha}$ and time spent in hypoglycaemia (over $24 \mathrm{~h}$ period). Circles, CON; crosses, CWT; triangles, IWT. Numbers indicate the unique participant identification number

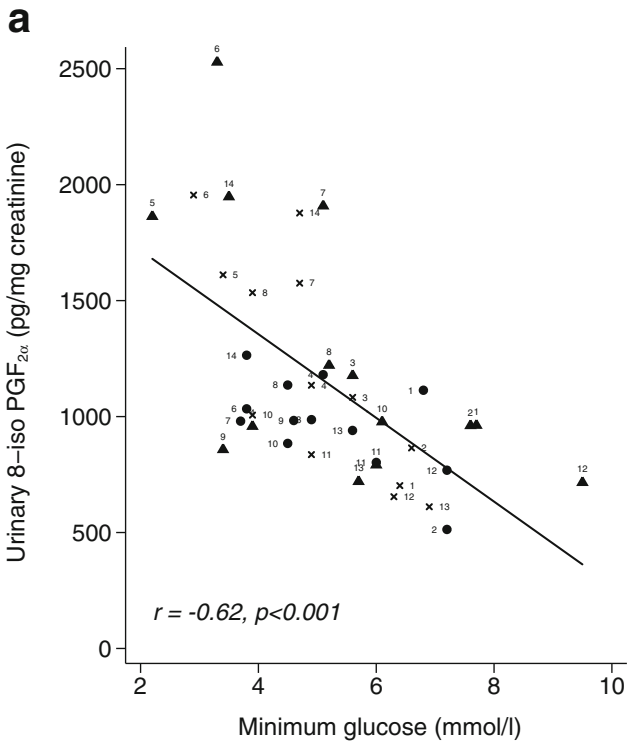

[3, 29, 31], others have reported positive effects for CWT-like interventions $[32,33]$. Since adherence to the training regime was very high and both the training duration and intensity was well controlled, we speculate that the peak exercise intensity during CWT was not high enough to elicit positive changes [34].

We did not find any significant associations between baseline values/changes in MAGE, mean CGM glucose levels or maximum CGM glucose levels and baseline values/changes in 8-iso $\mathrm{PGF}_{2 \alpha}$. The lack of association between MAGE and 8-iso $\mathrm{PGF}_{2 \alpha}$ is especially surprising since Monnier et al demonstrated a strong relationship between these two variables in individuals with type 2 diabetes [16]. The reason for this discrepancy is unclear; compared with the study by Monnier et al, MAGE was assessed using the same method and the ELISA kits used to measure 8-iso $\mathrm{PGF}_{2 \alpha}$ were from the same company. Despite the gold standard for measuring 8 -iso $\mathrm{PGF}_{2 \alpha}$ being mass spectrometry [35], a comparison between mass spectrometry and enzyme immunoassay-based measurement of 8 -iso $\mathrm{PGF}_{2 \alpha}$ has revealed a good correlation between the two methods $(r=0.80$ [36]). One difference between the studies is that our mean baseline MAGE levels were substantially higher than those reported by Monnier et al $(6.2 \pm 0.3 \mathrm{mmol} / \mathrm{l}$ vs $4.2 \pm 0.3 \mathrm{mmol} / \mathrm{l})$ and, although no data are available, it might be speculated that the linear relationship between MAGE and 8-iso $\mathrm{PGF}_{2 \alpha}$ disappears with higher glycaemic variability. The difference in the number of individuals analysed in the two studies (13 vs 21) may also contribute to this discrepancy. Moreover, it must be emphasised that there are other studies that have not been able to demonstrate the close association between MAGE and 8-iso $\mathrm{PGF}_{2 \alpha}[37,38]$.

Exploratory analyses indicated an inverse relationship between 8-iso $\mathrm{PGF}_{2 \alpha}$ and minimum glucose levels. Moreover, time spent in hypoglycaemia (glucose $<4.0 \mathrm{mmol} / \mathrm{l}$ ) was positively correlated to 8 -iso $\mathrm{PGF}_{2 \alpha}$. These associations are highly clinically relevant, given the suggested relationship between oxidative stress and cardiovascular disease [14]. However, it must be noted that CGM measurements in the hypoglycaemic range are somewhat inaccurate [39] and therefore our findings need to be confirmed in a controlled setting. The causative role of hypoglycaemia in oxidative stress is lent support by a study in which a clamp-based investigation in individuals with type 1 diabetes showed that while hyperglycaemia itself causes oxidative stress, the increase in oxidative stress levels are much higher if the hyperglycaemia is preceded by hypoglycaemia [17]. The authors concluded that the body is more vulnerable to hyperglycaemia when it has recently experienced hypoglycaemia. Studies examining whether this pattern is also seen in individuals with type 2 diabetes are needed.

None of the interventions in the present study resulted in a change in systemic oxidative stress levels, and we did not find any significant associations between changes in glycaemic control variables and changes in oxidative stress, either when evaluating all interventions or within any of the interventions. In that context, it should be noted that exercise may acutely elevate 8-iso $\mathrm{PGF}_{2 \alpha}$ [40]. Whereas this exercise-induced elevation is typically considered to be short-lived and therefore not affecting measurements starting $15 \mathrm{~h}$ after cessation of the exercise bout [35], we cannot rule out that a potential traininginduced reduction was outbalanced by a potential acute exercise-induced elevation in 8-iso $\mathrm{PGF}_{2 \alpha}$. Additionally, since diabetes is a condition wherein chronically elevated oxidative stress levels develop over years [14], possibly more than 2 weeks of exercise training is required to remedy this. Since 4 months of IWT has been shown to improve glycaemic control when compared with CWT [3], and because of the close association between systemic oxidative stress and inflammation [15] and the indirect anti-inflammatory effects of exercise 
as a result of training-induced improvements in body composition [41], studies of longer duration are warranted to further examine the potential effects of exercise on oxidative stress.

One limitation of the study is that, although no significant baseline differences between interventions were seen in any variables measured, we did see numerically higher baseline levels for IWT compared with CWT and CON (Table 1). In particular, CGM-derived measures of glycaemic control were considerably higher at baseline for CON compared with baseline for IWT. While the reason for this is unknown, it may have resulted in regression towards the mean, which may have potentiated the effects seen. However, although post hoc analyses adjusted for the respective baseline values did decrease some of the effect sizes, all significant improvements in the CGM-derived variables observed with IWT were still present after adjustments (data not shown).

Another limitation is the small sample size for this study. As such, effect sizes in small studies are often greater than in larger studies [42]. Also, since the apparent baseline differences in variables potentially may have been influenced by the small sample size, our findings should be confirmed in a larger study.

In summary, 2 weeks of IWT, but not oxygen-consumptionmatched CWT, improves CGM-derived measures of glycaemic control, including glycaemic variability, independent of changes in body composition and physical fitness. Although these findings should be confirmed using a larger number of participants, they may have implications for the way in which future training programmes are structured and recommended for individuals with type 2 diabetes.

Acknowledgements R. Rovsing, H. Villumsen, M. Gamst Høiberg, L. Kjølbo, S. H. Knudsen, L. Seier Hansen, S. Thorsen and C. Køllner Olsen (all affiliated to The Centre for Physical Activity Research [CFAS], Rigshospitalet, Copenhagen, Denmark) are acknowledged for their technical assistance.

Data availability The data that support the findings of this study are available on request from the corresponding author (KK), given that this does not violate the laws of the Danish Data Protection Agency.

Funding The Centre for Physical Activity Research (CFAS) is supported by a grant from TrygFonden. During the study period, the Centre of Inflammation and Metabolism (CIM) was supported by a grant from the Danish National Research Foundation (DNRF55). The study was further supported by grants from Diabetesforeningen, Augustinusfonden and Krista og Viggo Petersens Fond. CIM/CFAS is a member of the Danish Center for Strategic Research in Type 2 Diabetes (DD2; the Danish Council for Strategic Research, grant no. 09-067009 and 09-075724). MR-L was supported by a post-doctoral grant from the Danish Diabetes Academy supported by the Novo Nordisk Foundation.

Duality of interest The authors declare that there is no duality of interest associated with this manuscript.
Contribution statement $\mathrm{KK}$ designed the study and obtained the funding. TPJS and BKP contributed to the study design. KK, MAC, IJ and IAM acquired the data. KK, MAC and MR-L analysed the data. KK wrote the manuscript. All authors reviewed and revised the manuscript and approved the final version. KK is responsible for the integrity of the work, as a whole.

\section{References}

1. Colberg SR, Sigal RJ, Fernhall B et al (2010) Exercise and type 2 diabetes: the American College of Sports Medicine and the American Diabetes Association: joint position statement. Diabetes Care 33:e147-e167

2. Gibala MJ, Little JP, Macdonald MJ, Hawley JA (2012) Physiological adaptations to low-volume, high-intensity interval training in health and disease. J Physiol 590:1077-1084

3. Karstoft K, Winding K, Knudsen SH et al (2013) The effects of free-living interval-walking training on glycemic control, body composition, and physical fitness in type 2 diabetes patients. Diabetes Care 36:228-236

4. Tjonna AE, Lee SJ, Rognmo O et al (2008) Aerobic interval training versus continuous moderate exercise as a treatment for the metabolic syndrome: a pilot study. Circulation 118:346-354

5. Mitranun W, Deerochanawong C, Tanaka H, Suksom D (2014) Continuous vs interval training on glycemic control and macroand microvascular reactivity in type 2 diabetic patients. Scand J Med Sci Sports 24:69-76

6. Robinson E, Durrer C, Simtchouk S et al (1985) Short-term highintensity interval and moderate-intensity continuous training reduce leukocyte TLR4 in inactive adults at elevated risk of type 2 diabetes. J Appl Physiol 119:508-516

7. Coker RH, Williams RH, Yeo SE et al (2009) The impact of exercise training compared to caloric restriction on hepatic and peripheral insulin resistance in obesity. J Clin Endocrinol Metab 94:4258 4266

8. Ross R, Dagnone D, Jones PJ et al (2000) Reduction in obesity and related comorbid conditions after diet-induced weight loss or exercise-induced weight loss in men. A randomized, controlled trial. Ann Intern Med 133:92-103

9. Irving BA, Davis CK, Brock DW et al (2008) Effect of exercise training intensity on abdominal visceral fat and body composition. Med Sci Sports Exerc 40:1863-1872

10. Tremblay A, Simoneau JA, Bouchard C (1994) Impact of exercise intensity on body fatness and skeletal muscle metabolism. Metabolism 43:814-818

11. Boule NG, Haddad E, Kenny GP, Wells GA, Sigal RJ (2001) Effects of exercise on glycemic control and body mass in type 2 diabetes mellitus: a meta-analysis of controlled clinical trials. JAMA 286:1218-1227

12. Shaw K, Gennat H, O Rourke P, Del MC (2006) Exercise for overweight or obesity. Cochrane Database Syst Rev, Issue 4 Art. no.: CD003817. doi: 10.1002/14651858.CD003817.pub3

13. Schramm TK, Gislason GH, Kober L et al (2008) Diabetes patients requiring glucose-lowering therapy and nondiabetics with a prior myocardial infarction carry the same cardiovascular risk: a population study of 3.3 million people. Circulation 117:1945-1954

14. Giacco F, Brownlee M (2010) Oxidative stress and diabetic complications. Circ Res 107:1058-1070

15. Salzano S, Checconi P, Hanschmann EM et al (2014) Linkage of inflammation and oxidative stress via release of glutathionylated 
peroxiredoxin-2, which acts as a danger signal. Proc Natl Acad Sci U S A 111:12157-12162

16. Monnier L, Mas E, Ginet $\mathrm{C}$ et al (2006) Activation of oxidative stress by acute glucose fluctuations compared with sustained chronic hyperglycemia in patients with type 2 diabetes. JAMA 295: 1681-1687

17. Ceriello A, Novials A, Ortega E et al (2012) Evidence that hyperglycemia after recovery from hypoglycemia worsens endothelial function and increases oxidative stress and inflammation in healthy control subjects and subjects with type 1 diabetes. Diabetes 61: 2993-2997

18. Stratton IM, Adler AI, Neil HA et al (2000) Association of glycaemia with macrovascular and microvascular complications of type 2 diabetes (UKPDS 35): prospective observational study. BMJ 321:405-412

19. Su G, Mi S, Tao H et al (2011) Association of glycemic variability and the presence and severity of coronary artery disease in patients with type 2 diabetes. Cardiovasc Diabetol 10:19

20. Mikus CR, Oberlin DJ, Libla J, Boyle LJ, Thyfault JP (2012) Glycaemic control is improved by 7 days of aerobic exercise training in patients with type 2 diabetes. Diabetologia 55:1417-1423

21. Little JP, Gillen JB, Percival M et al (2011) Low-volume highintensity interval training reduces hyperglycemia and increases muscle mitochondrial capacity in patients with type 2 diabetes. J Appl Physiol 111:1554-1560

22. Ryden L, Grant PJ, Anker SD et al (2013) ESC Guidelines on diabetes, pre-diabetes, and cardiovascular diseases developed in collaboration with the EASD: the Task Force on diabetes, pre-diabetes, and cardiovascular diseases of the European Society of Cardiology (ESC) and developed in collaboration with the European Association for the Study of Diabetes (EASD). Eur Heart J 34:3035-3087

23. Pedersen BK, Saltin B (2015) Exercise as medicine - evidence for prescribing exercise as therapy in 26 different chronic diseases. Scand J Med Sci Sports 25(Suppl 3):1-72

24. Taylor HL, Jacobs DR Jr, Schucker B, Knudsen J, Leon AS, Debacker G (1978) A questionnaire for the assessment of leisure time physical activities. J Chronic Dis 31:741-755

25. Borg GA (1982) Psychophysical bases of perceived exertion. Med Sci Sports Exerc 14:377-381

26. Mifflin MD, St Jeor ST, Hill LA, Scott BJ, Daugherty SA, Koh YO (1990) A new predictive equation for resting energy expenditure in healthy individuals. Am J Clin Nutr 51:241-247

27. Seaquist ER, Anderson J, Childs B et al (2013) Hypoglycemia and diabetes: a report of a workgroup of the American Diabetes Association and the Endocrine Society. Diabetes Care 36:13841395

28. Service FJ, Molnar GD, Rosevear JW, Ackerman E, Gatewood LC, Taylor WF (1970) Mean amplitude of glycemic excursions, a measure of diabetic instability. Diabetes 19:644-655
29. Karstoft K, Winding K, Knudsen SH et al (2014) Mechanisms behind the superior effects of interval vs continuous training on glycaemic control in individuals with type 2 diabetes: a randomised controlled trial. Diabetologia 57:2081-2093

30. Zierath JR (2002) Invited review: exercise training-induced changes in insulin signaling in skeletal muscle. J Appl Physiol 93:773781

31. Karstoft K, Christensen CS, Pedersen BK, Solomon TP (2014) The acute effects of interval- vs. continuous-walking exercise on glycemic control in subjects with type 2 diabetes: a cross-over, controlled study. J Clin Endocrinol Metab 99:3334-3342

32. Walker KZ, Piers LS, Putt RS, Jones JA, O Dea K (1999) Effects of regular walking on cardiovascular risk factors and body composition in normoglycemic women and women with type 2 diabetes. Diabetes Care 22:555-561

33. Negri C, Bacchi E, Morgante S et al (2010) Supervised walking groups to increase physical activity in type 2 diabetic patients. Diabetes Care 33:2333-2335

34. Liubaoerjijin Y, Terada T, Fletcher K, Boule NG (2016) Effect of aerobic exercise intensity on glycemic control in type 2 diabetes: a meta-analysis of head-to-head randomized trials. Acta Diabetol 53: 769-781

35. Nikolaidis MG, Kyparos A, Vrabas IS (2011) $\mathrm{F}_{2}$-isoprostane formation, measurement and interpretation: the role of exercise. Prog Lipid Res 50:89-103

36. Devaraj S, Hirany SV, Burk RF, Jialal I (2001) Divergence between LDL oxidative susceptibility and urinary $\mathrm{F}_{2}$-isoprostanes as measures of oxidative stress in type 2 diabetes. Clin Chem 47:1974 1979

37. Siegelaar SE, Kulik W, van Lenthe H, Mukherjee R, Hoekstra JB, DeVries JH (2009) A randomized clinical trial comparing the effect of basal insulin and inhaled mealtime insulin on glucose variability and oxidative stress. Diabetes Obes Metab 11:709-714

38. Di FA, Picconi F, Di SP et al (2011) Impact of glycemic and blood pressure variability on surrogate measures of cardiovascular outcomes in type 2 diabetic patients. Diabetes Care 34:1605-1609

39. Bay C, Kristensen PL, Pedersen-Bjergaard U, Tarnow L, Thorsteinsson B (2013) Nocturnal continuous glucose monitoring: accuracy and reliability of hypoglycemia detection in patients with type 1 diabetes at high risk of severe hypoglycemia. Diabetes Technol Ther 15:371-377

40. Rietjens SJ, Beelen M, Koopman R, van Loon LJ, Bast A, Haenen GR (2007) A single session of resistance exercise induces oxidative damage in untrained men. Med Sci Sports Exerc 39:2145-2151

41. Karstoft K, Pedersen BK (2016) Exercise and type 2 diabetes: focus on metabolism and inflammation. Immunol Cell Biol 94:146-150

42. Button KS, Ioannidis JP, Mokrysz C et al (2013) Power failure: why small sample size undermines the reliability of neuroscience. Nat Rev Neurosci 14:365-376 